\title{
Inductive effect of methyl group in a series of methylated indoles: A graph theoretical analysis in the light of density functional theory and correlation with experimental charge transfer transition energies
}

\author{
AMIT S TIWARY,* and ASOK K MUKHERJEE ${ }^{\mathrm{b}}$ \\ ${ }^{a}$ Department of Chemistry, Netaji Mahavidyalaya, Arambagh, Hooghly 712 601, India \\ ${ }^{b}$ Digital Computer System, 112, R B Ghosh Road, Burdwan 713 101, India \\ e-mail: amitstiwary@gmail.com
}

MS received 24 June 2012; revised 21 October 2012; accepted 17 December 2012

\begin{abstract}
The inductive effect of methyl group has been quantified by expressing highest occupied molecular orbital (HOMO) and HOMO-1 energies of indole and a series of methylated indoles using a combination of graph theory (GT) and the Coulson-Longuett-Higgins perturbation method. By correlating these expressions with the corresponding Kohn-Sham orbital energies of the indoles obtained by density functional theory (DFT) calculation at the B3LYP/6-31++G(d,p) and M06-2X/6-31++G(d,p) levels of theory, the inductive effect parameter $h_{M e}$ has been estimated; the Coulomb integral $\alpha$ of $\pi$-conjugated carbon atom also comes out from the analysis. A correlation of the GT results with the HOMO and HOMO-1 energies obtained by the HF/STO3G method yield almost the same values of $h_{M e}$ and $\alpha$. Finally, when these estimated $h_{M e}$ and $\alpha$ are used to calculate the vertical ionization potentials of the methylated indoles in the series, an excellent correlation with experimental charge transfer transition energies of their molecular complexes with tetracyanoethylene is obtained which complies with Mulliken's theory of charge transfer.
\end{abstract}

Keywords. Graph theory; DFT; inductive effect; methyl indoles; charge transfer.

\section{Introduction}

Ab initio quantum mechanical methods and those based on the density functional theory (DFT) $)^{1,2}$ are currently used for investigation of molecular structure, spectroscopic and other physicochemical properties and reaction mechanisms. ${ }^{3-9}$ On the other hand, methods of graph theory (GT) are useful, particularly for large $\pi$ conjugated systems, owing to the fact that the adjacency matrix of the hydrogen-suppressed molecular graph is the same as the Hückel Hamiltonian matrix of the system, with proper scaling of energy (Coulomb integral, $\alpha=0$ and resonance integral, $\beta=$ unit of energy). The Hückel Molecular Orbital (HMO) theory, ${ }^{10}$ despite being a naive $\pi$-electron theory, has not lost its utility or relevance. ${ }^{11-21}$ Thus, DFT methods have been shown ${ }^{11}$ to yield rules for $3 \mathrm{D}$ aromatic hydrogen clusters analogous to the Hückel rule for planar aromatic systems. An interaction between Lewis configurations and Hückel energies have shown ${ }^{12}$ to provide a useful alternative to sophisticated computational procedures for assessing the weights of the Lewis structures involved in mesomery. Dias and Guirgis have

*For correspondence shown ${ }^{13}$ that embedding and right-hand mirror plane fragments (Hall ${ }^{14}$ and McClelland ${ }^{15}$ subgraphs, respectively) of graphs representing $\pi$-conjugated molecules are Molecular Orbital (MO) functional groups of importance in molecular modelling of near-infrared absorbing pigments and the GT technique used in this work circumvents the need for a full molecule calculation. Study of dendralene and radialene hydrocarbons in terms of reciprocal graphs ${ }^{16}$ has been shown ${ }^{17}$ to be important in the design of conducting and ferromagnetic polymers. GT methods are particularly useful for streamlining HMO calculations for large $\pi$-networks. ${ }^{18}$ Thus, by applying GT methods antiaromatic holes and related defects in graphenes have been analysed ${ }^{19}$ and Schottky defects in graphenes have been modelled ${ }^{20}$ in terms of concealed coronoid hydrocarbons with enhanced antiaromatic circuit contributions. A simple topological method has been developed ${ }^{21}$ for estimating the superaromatic stabilization energy of a superring molecule. The perturbation method introduced by Coulson and Longuet-Higgins ${ }^{22}$ in the context of HMO formalism is a powerful and conceptually rich tool in understanding observed sequences in many spectroscopic and reaction mechanistic studies. Such calculations can be streamlined by use of GT. To achieve 
compatibility between GT and DFT results, it is desirable to find the perturbational HMO parameters from DFT-calculated orbital energies. The objective of the present work is to find the parameter $h_{M e}$ for the inductive effect of methyl group and also to evaluate the Coulomb integral $\alpha$ of the $\pi$-conjugated carbon atom of HMO theory by correlating the DFT-calculated energies of the occupied Kohn-Sham (KS) orbitals of a series of methylated indoles with their graph theoretically calculated values. A correlation with the highest occupied molecular orbital (HOMO) and HOMO-1 energies of the indoles calculated by the HF/STO-3G method will also be performed for estimating the $h_{M e}$ and $\alpha$ parameters and comparing them with their values obtained from GT-DFT correlation. Finally, the reliability of the estimated values of these two parameters will be tested by correlating the GT-calculated HOMO energies (using these parameters) with the charge transfer (CT) transition energies of the molecular complexes of these indoles with Tetracyanoethylene (TCNE) in the light of Mulliken's theory. ${ }^{23,24}$

\section{Calculation of the $\pi$-MO energies of methylindoles in terms of $h_{M e}$ using graph theoretical method}

When a methyl group (Me-) is attached to a $\pi$ conjugated atom, the perturbation caused by the inductive effect of Me- changes the Coulomb integral of that conjugated atom. The energy of the $j$ th $\pi-\mathrm{MO}$ of a methylated indole is given, according to the CoulsonLonguet-Higgins perturbation method ${ }^{22}$ in HMO formalism, by

$$
E_{j}=\alpha+x_{j}^{0} \beta+h_{M e} \beta \sum_{r} C_{r j}^{2},
$$

where $\alpha$ and $\beta$ are Coulomb and resonance integrals of $\mathrm{sp}^{2}-\mathrm{C}$ atom and $\mathrm{C}\left(\mathrm{sp}^{2}\right)-\mathrm{C}\left(\mathrm{sp}^{2}\right)$ bond respectively; $x_{j}^{0}$ is the energy of the $j$ th $\pi$-MO of the unperturbed system, indole, and $r$ is the location of the methyl group in the heterocyclic ring. The parameter $h_{M e}$ is defined by

$$
\alpha^{\prime}=\alpha+h_{M e} \beta,
$$

where $\alpha^{\prime}$ is Coulomb integral of the atom of the indole ring to which the methyl group is attached. In GT the $\mathrm{sp}^{2}-\mathrm{C}$ atoms are denoted by vertices and $\mathrm{C}-\mathrm{C}$ bonds by edges. The heteroatoms $(X)$ in the $\pi$-conjugated system are denoted by weighted vertices and $\mathrm{C}-\mathrm{X}$ bonds by weighted edges. The perturbational parameters $h_{X}$ and $k_{C-X}$ act respectively as the vertex- and edgeweights. For the present purpose the hydrogen suppressed molecular graph of indole is the vertex- and edge-weighted graph, $G_{x}$ and the necessary subgraphs for construction of its characteristic polynomial (CP) are (i) $G_{x}-v_{x}$ (obtained by deleting from $G_{x}$ the vertex $v_{x}$ which represents the heteroatom, N) and (ii) the isoconjugate graph, $G$ (figure 1). The $\mathrm{CP}$ of $G_{x}$ is given ${ }^{25}$ by

$$
\begin{aligned}
P\left(G_{x} ; X\right)= & (X-h) P\left(G_{x}-v_{x} ; X\right) \\
& -k^{2} X\left[P\left(G_{x}-v_{x} ; X\right)-P(G ; X)\right] .
\end{aligned}
$$

Here for simplicity we have written $h$ and $k$, respectively for $h_{N}$ and $k_{C-N}$. All the subgraphs on the right hand side of equation (3) can be subjected to McClelland's method ${ }^{15}$ of mirror plane factorization to get linear chains as fragments (figure 2). Of these, the fragment (B) is simply a $\mathrm{K}_{2}$ subgraph having $\mathrm{CP}=$ $\left(x^{2}-1\right)$. For the other linear chains it is convenient to use the tabular method developed ${ }^{26}$ and used earlier. ${ }^{27}$ Construction of the CPs of these chains is shown in tables 1,2 and 3 .
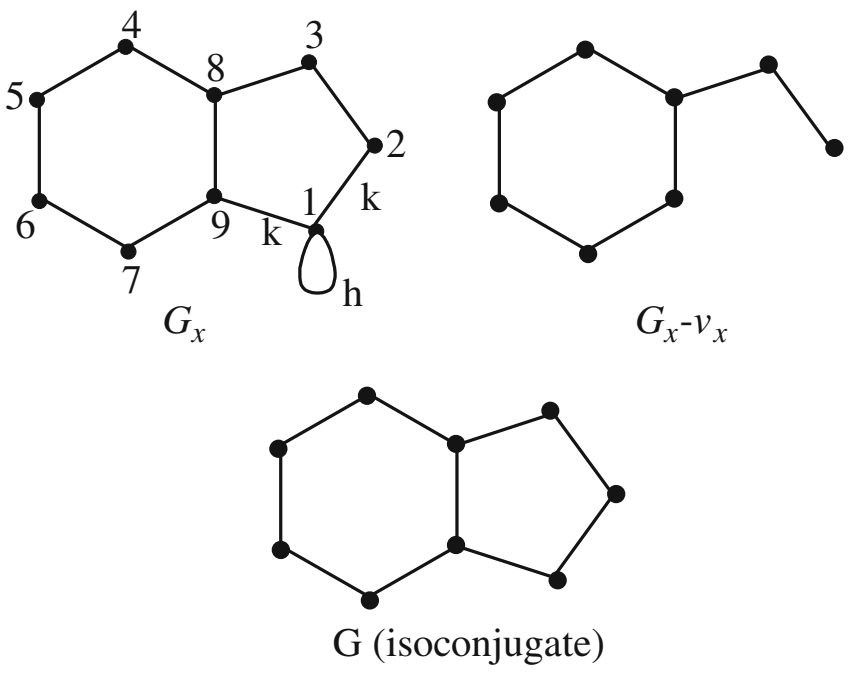

Figure 1. Molecular graph of indole $\left(G_{x}\right)$, and the subgraphs necessary for calculation of its $\mathrm{CP}$.
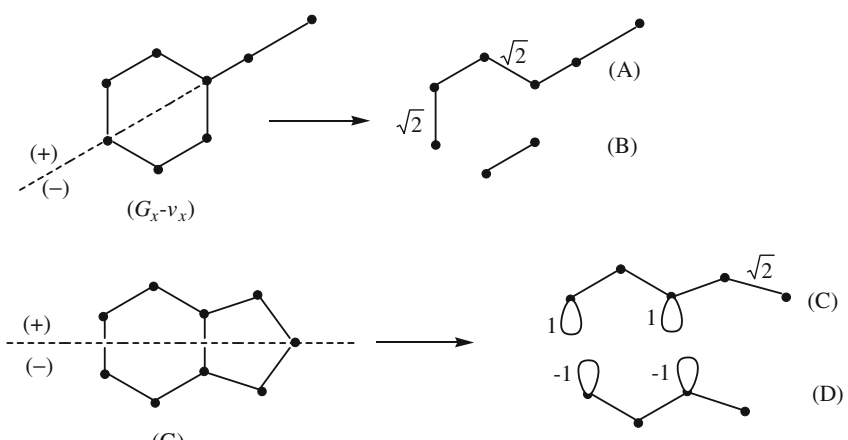

(G)

Figure 2. Mirror plane factorization of $G_{x}-v_{x}$ and the isoconjuage graph $\mathrm{G}$. 
Table 1. Construction of $\mathrm{CP}$ of the linear chain (A).

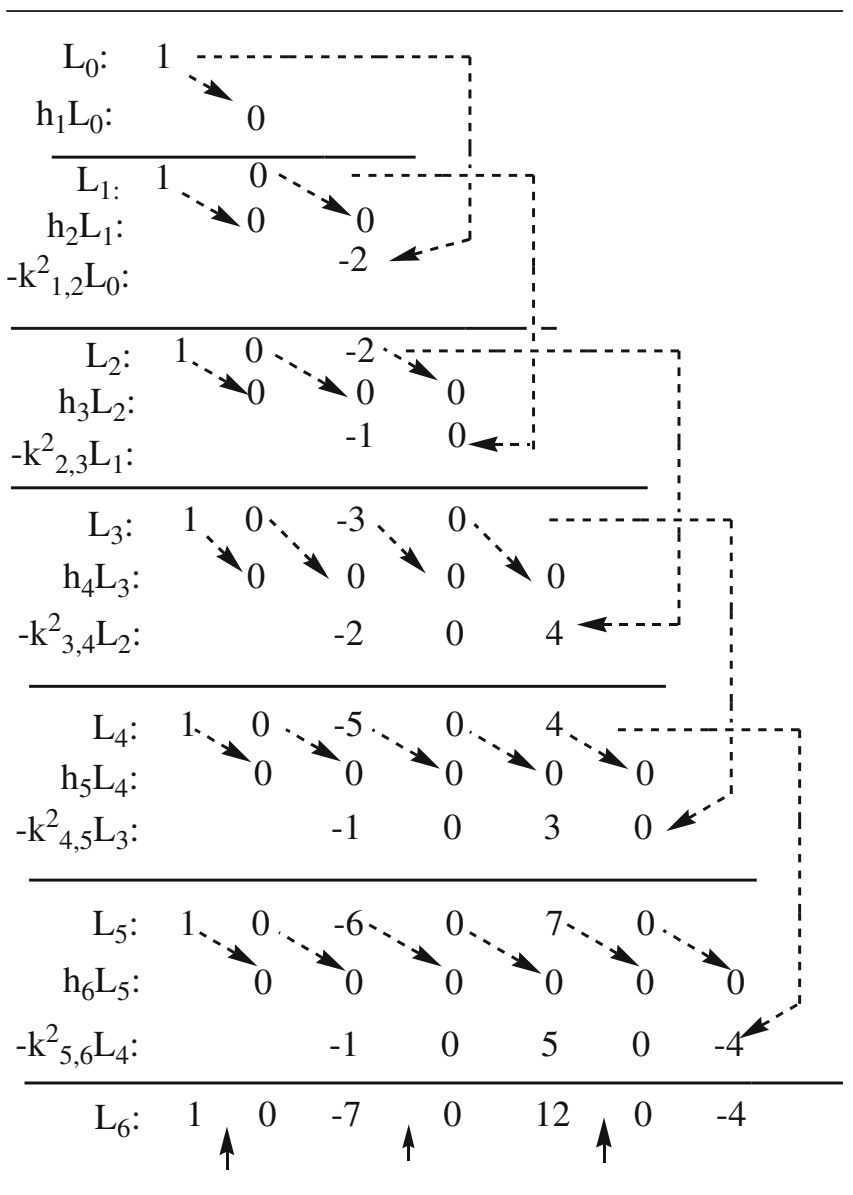

Table 2. Construction of $\mathrm{CP}$ of the linear chain (C).

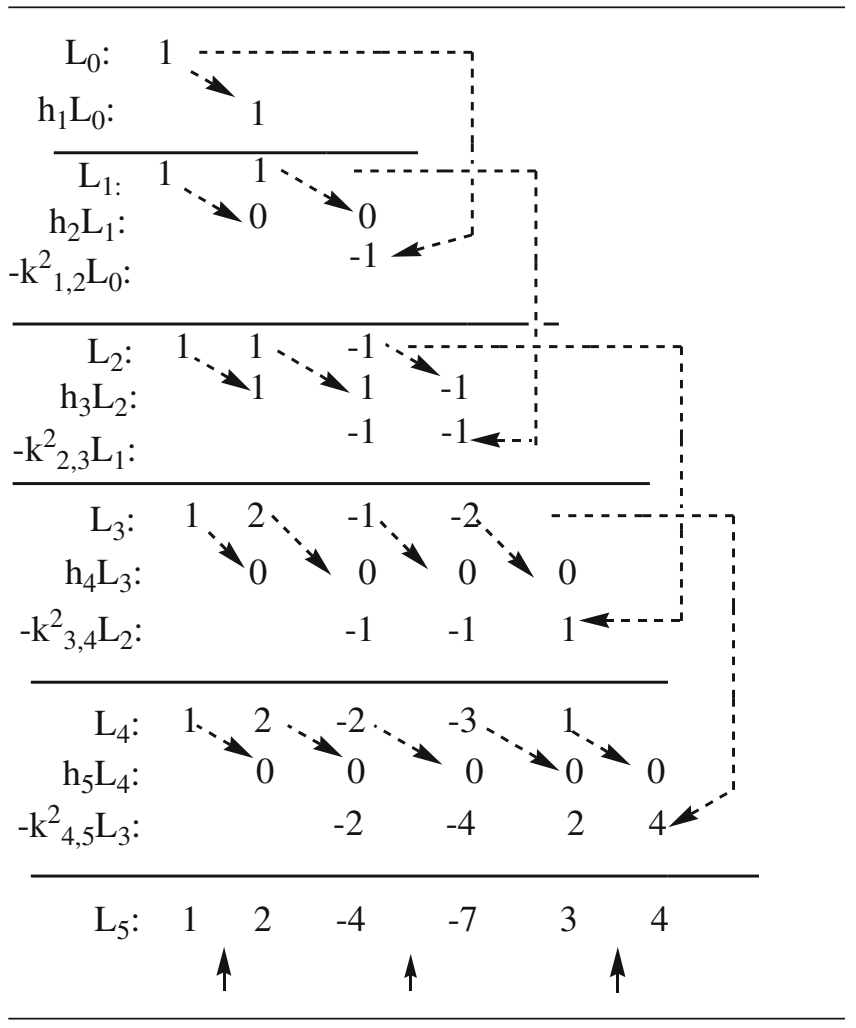

Table 3. Construction of CP of the linear chain (D).

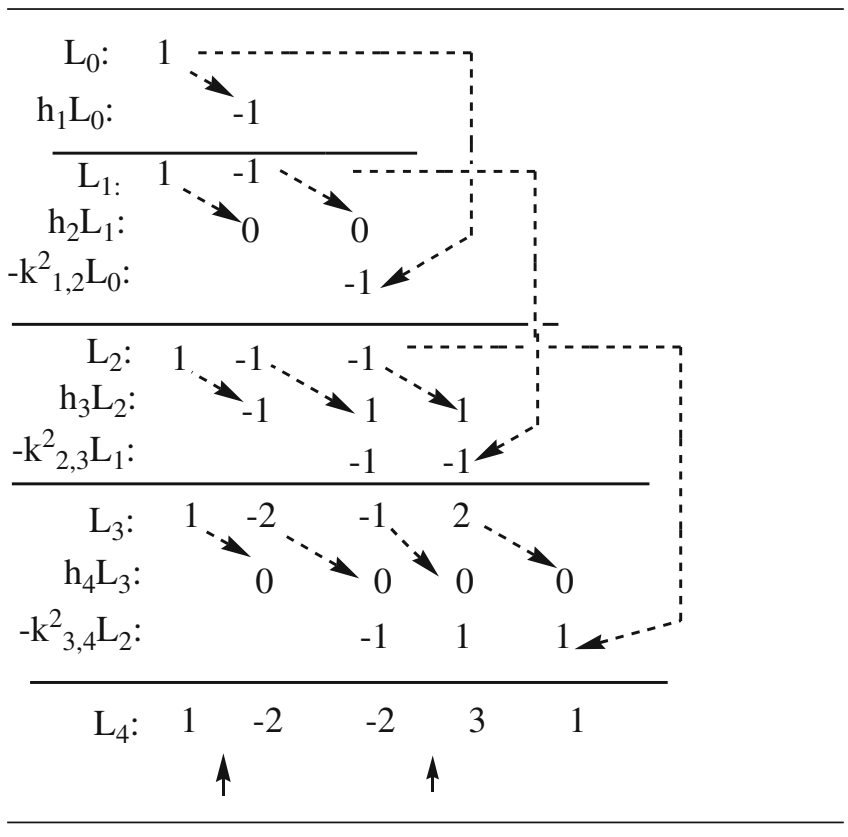

From these tables we have, using eq. (3),

$$
\begin{aligned}
P\left(G_{x} ; X\right)= & (X-h)\left(X^{2}-1\right)\left(X^{6}-7 X^{4}+12 X^{2}-4\right) \\
& -k^{2} X\left(X^{2}-1\right)\left(X^{6}-7 X^{4}+12 X^{2}-4\right) \\
& +k^{2}\left(X^{5}-2 X^{4}-4 X^{3}+7 X^{2}+3 X-4\right) \\
& \times\left(X^{4}+2 X^{3}-2 X^{2}-3 X+1\right) .
\end{aligned}
$$

Streitwieser's recommended value ${ }^{28}$ for nitrogen in aza-aromatics is in the range $h_{N}=1.0$ to 1.5 and $k_{C N}=$ 0.8 to 1.0. From eq. (4), we have, using $h_{N}=1.0$ and $k_{C N}=1.0$ (for $h$ and $k$, respectively) the characteristic polynomial for indole:

$$
\begin{aligned}
P\left(G_{x} ; X\right)= & X^{9}-X^{8}-10 X^{7}+8 X^{6}+32 X^{5} \\
& -21 X^{4}-39 X^{3}+22 X^{2}+15 X-8
\end{aligned}
$$

The highest positive root of $P\left(G_{x} ; X\right)=0$ can be easily obtained by using the Budan-Fourier theorem ${ }^{29}$ followed by Newton-Raphson iteration. The theorem states that for an n-degree polynomial $P(x)$, all the zeros of which are real, the number of zeros lying in the range (a,b), a $<\mathrm{b}$, is equal to $N(\mathrm{a})-N(\mathrm{~b})$, where $N(t)$, $t=a$ and $b$, is equal to the number of sign-changes in the sequence $P(t), P^{(1)}(t), P^{2}(t), P^{3}(t), \ldots \ldots, P^{n}(t)$ with $P^{k}(t)$ denoting the $k$ th derivative of $P(X)$ at $X=$ $t$. In the present case with $P\left(G_{x} ; X\right)$ and its nine derivatives, we have, at $X=0,0.5$ and 0.55 , the following sign sequences:

$$
\begin{aligned}
& X=0:[-++--++--+], N(0)=5 \\
& X=0.5:[-+--++--++], N(0.5)=5 \\
& X=0.55:[++-++---++], N(0.55)=4
\end{aligned}
$$


Hence the polynomial $P\left(G_{x} ; X\right)$ has no zero in the range $(0,0.5)$ and exactly one $(5-4=1)$ zero in $(0.5,0.55)$. Now starting with 0.55 and applying the Newton-Raphson method on the equation $P\left(G_{x} ; X\right)=$ 0 one gets convergence at $X=0.52014$ after only two iterations. This is the highest positive eigenvalue of the graph $G_{x}$. We call it $X_{5}$, after arranging the eigenvalues of $G_{x}$ in the decreasing sequence: $X_{1}, X_{2}, X_{3}, \ldots \ldots$, $X_{9}$. These are the $\pi$-MO energies of indole in units of $\beta$, with $\alpha$ as the zero level of energy. The value of $C_{r 5}^{2}$, required for perturbational coefficients, can now be obtained by the Ulam subgraph method: ${ }^{30}$

$$
C_{r 5}^{2}=\frac{P\left(G_{x}-v_{r} ; X\right)}{d\left[P\left(G_{x} ; X\right)\right] / d X} \quad \text { at } X=X_{5},
$$

where $G_{x}-v_{r}$ is the Ulam $^{31}$ subgraph obtained by deleting the vertex $v_{r}$ from $G_{x}$. For $C_{15}^{2}$ one required
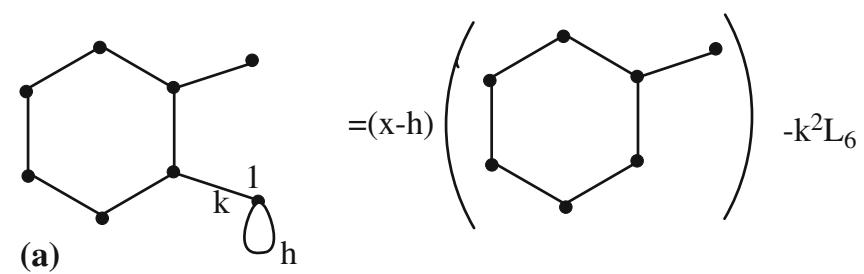

(a)

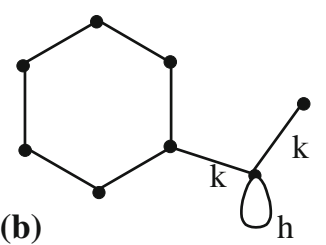

(b)

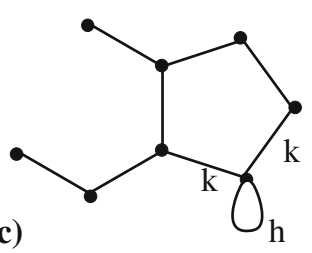

Figure 3. Ulam subgraphs necessary for calculation of $\mathrm{C}_{\mathrm{r} 5}^{2}$.
Ulam subgraph is $G_{x}-v_{x}$ whose $\mathrm{CP}$ is constructed and shown in table 1 . The other necessary Ulam subgraphs are shown in figure 3 . Values of $C_{r 5}^{2}$ calculated in this way are shown in table 4. In the same way the HOMO1 level, $X_{4}$, was found to be 0.792335 and the perturbational coefficients $C_{r 4}^{2}$ for the indoles were calculated using eq. (7) taking $X=X_{4}$ and are given in table 4 .

\section{DFT-calculated energies of the highest occupied and the next lower KS orbitals of the methylated indoles}

The energies of the highest occupied and the next lower KS orbitals of indole and the methylated indoles were calculated on the basis of DFT. ${ }^{1,2}$ These two orbitals will henceforth be denoted by HOKSO and HOKSO-1, respectively. The combination of Becke's three-parameter hybrid ${ }^{32}$ exchange potential with the correlation functional of Lee, Yang and $\operatorname{Parr}^{33}$ (B3LYP) was used in the calculation. The geometries of indole and the methylated indoles were optimized in vacuo and the KS orbital energies were calculated with these optimized geometries using the $6-31++\mathrm{G}(\mathrm{d}, \mathrm{p})$ basis set. All the computations were performed on a Pentium computer with the Gaussian 03 Revision-D.01 suite of programmes. ${ }^{34}$ Results are given in table 4 . That the DFT-calculated HOMOs and the HOMO-1 orbitals for the indoles are really $\pi$-MOs, can be ensured by inspection of the nodal planes of the latter. Two sample Gauss View pictures, one for the DFT calculated HOKSO and the other for HOKSO-1 of 2,5-dimethyl indole are shown in figures 4 and 5 wherefrom their $\pi$-MO character is evident. Again, since the relatively recent Truhler ${ }^{35}$ functionals are known to take into account weak interactions (dispersion effects in particular) better, ${ }^{36-39}$ calculations were also performed at the M062X/6-31++G(d,p) level using the Gaussian 09 suite

Table 4. DFT-calculated energy of HOKSO and HOKSO-1 and perturbational quantities $\sum_{r} C_{r 5}^{2}$ and $\sum_{r} C_{r 4}^{2}$ (calculated by the present GT method taking $h_{N}=1.0$, $\left.k_{C N}=1.0\right)$ of a series of methylated indoles.

\begin{tabular}{llllllll}
\hline & \multicolumn{2}{c}{$-E_{\mathrm{HOKSO}} / \mathrm{ha}$} & & \multicolumn{2}{c}{$-\left(E_{\mathrm{HOKSO}-1}\right) / \mathrm{ha}$} & & \\
\cline { 2 - 3 } Donor & $\mathrm{B} 3 \mathrm{LYP}$ & $\mathrm{M} 06-2 \mathrm{X}$ & & $\mathrm{B} 3 \mathrm{LYP}$ & $\mathrm{M} 06-2 \mathrm{X}$ & $\sum_{r} C_{r 5}^{2}$ & $\sum_{r} C_{r 4}^{2}$ \\
\hline Indole & 0.21091 & 0.25703 & 0.22754 & 0.27447 & 0 & 0 \\
1-Methyl indole & 0.20582 & 0.25163 & 0.22411 & 0.27077 & 0.1392 & 0.0790 \\
2-Methyl indole & 0.20518 & 0.25109 & 0.22170 & 0.26886 & 0.1095 & 0.1706 \\
3-Methyl indole & 0.20462 & 0.25065 & 0.22489 & 0.27195 & 0.2972 & 0.0021 \\
1,2-Dimethyl indole & 0.20068 & 0.24643 & 0.21808 & 0.26476 & 0.2487 & 0.2497 \\
2,3-Dimethyl indole & 0.19946 & 0.24517 & 0.21908 & 0.26632 & 0.4067 & 0.1729 \\
2,5-Dimethyl indole & 0.20245 & 0.24854 & 0.21360 & 0.26025 & 0.1384 & 0.3983
\end{tabular}




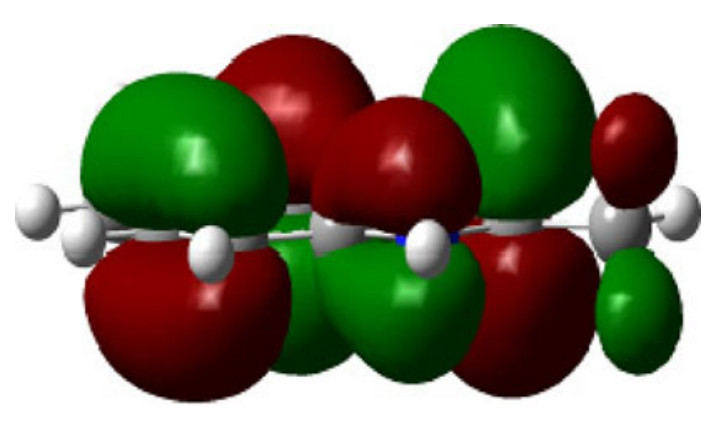

Figure 4. HOKSO of 2,5-dimethyl indole calculated at the DFT/B3LYP/6-31++G (d,p) level. The plane of the fused rings is the nodal plane which reveals the $\pi$-character of the orbital.

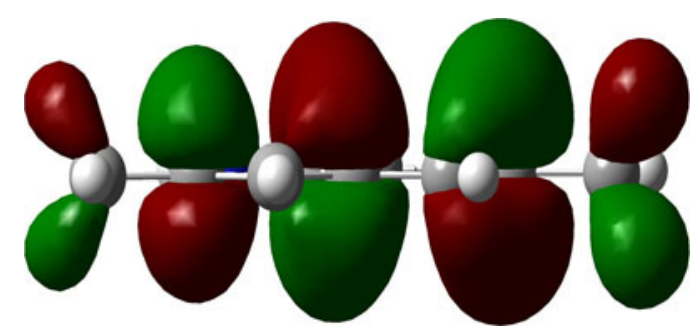

Figure 5. HOKSO-1 of 2,5-dimethyl indole calculated at the DFT/B3LYP/6-31++G (d,p) level. The plane of the fused rings is the nodal plane which reveals the $\pi$-character of the orbital.

of programmes. ${ }^{40}$ Results are given in table 4 along with the GT-calculated perturbational quantities, $\sum_{r} C_{r 4}^{2}$ and $\sum_{r} C_{r 4}^{2}$.

\section{Correlation of DFT-calculated energies} of HOKSO and (HOKSO-1) of the indoles with graph theoretically obtained $\sum_{r} C_{r 5}^{2}$ and $\sum_{r} C_{r 4}^{2}$, respectively

The HOKSO and HOKSO-1 energies calculated at the B3LYP/6-31++G(d,p) level and the perturbational quantities $\sum_{r} C_{r 5}^{2}$ and $\sum_{r} C_{r 5}^{2}$ for the series of indoles under consideration give excellent linear correlation as expected from eq. (1) and shown in figures 6 and 7. The following linear regression equations hold for the two energy levels:

$$
\begin{aligned}
E_{\mathrm{HOKSO}}= & (0.02227 \pm 0.00538) \sum_{r} C_{r 5}^{2} \\
& -(0.20885 \pm 0.00136) ; r=0.88
\end{aligned}
$$

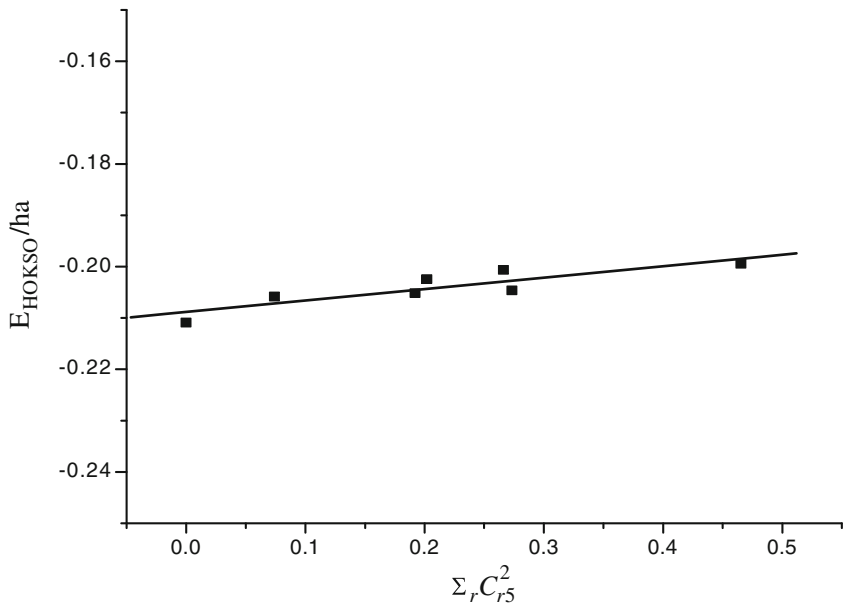

Figure 6. Plot of $E_{\mathrm{HOKSO}}$ against the perturbational coefficient for the indoles under study.

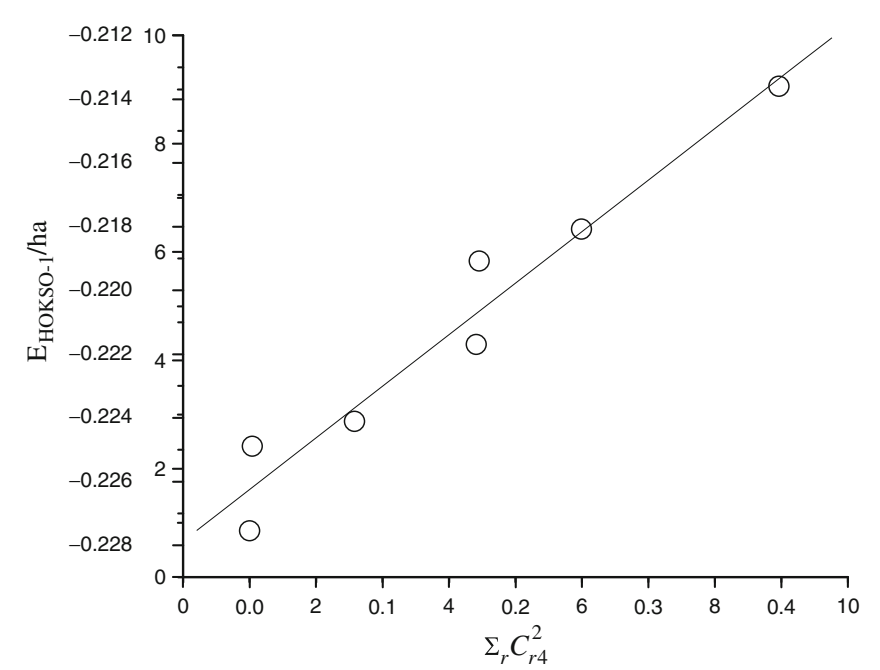

Figure 7. Plot of $E_{\mathrm{HOKSO}-1}$ against the perturbational coefficient for the indoles under study.

$$
\begin{aligned}
E_{\mathrm{HOKSO}-1}= & (0.03234 \pm 0.00339) \sum_{r} C_{r 4}^{2} \\
& +(-0.22624 \pm 0.00068) ; r=0.97,
\end{aligned}
$$

where the energies are in Hartree. Comparing these with eq. (1) and using $\beta=-2.39 \mathrm{eV}^{41}$ one finds

$h_{M e}=-0.25 \pm 0.06$ (from the slope of (7) and $-0.368 \pm 0.04$ from (8).

From the intercept of (7), $\alpha+x_{j}^{0} \beta=$ $\left(-0.20885\right.$ ha $\left.\times 27.2116 \mathrm{eV}-\mathrm{ha}^{-1} /-2.39 \mathrm{eV}\right) \quad \beta=$ $2.377884 \beta$. Now with $x_{j}^{0} \beta=0.52014 \beta$ (as found earlier in section 2) one finds $\alpha=(2.377884-0.52014)$ $\beta=-4.44 \mathrm{eV}$. Similarly from the intercept of (8) $\alpha$ is found to be $-4.26 \mathrm{eV}$. Average of these two, e.g., 
$-4.3 \mathrm{eV}$, is close to the one $(-4.5 \mathrm{eV})$ obtained in our earlier work with a series of phenols. ${ }^{27}$

With energies of the same two KS orbitals calculated at the M06-2X/6-31++G(d,p) level, given in table 4, the linear regression equations are:

$$
\begin{aligned}
E_{\mathrm{HOKSO}}= & (0.02387 \pm 0.00732) \sum_{r} C_{r 5}^{2} \\
& -(0.25464 \pm 0.00167) ; r=0.82 \\
E_{\mathrm{HOKSO}-1}= & (0.03301 \pm 0.00317) \sum_{r} C_{r 4}^{2} \\
& +(-0.27326 \pm 0.00064) ; r=0.98,
\end{aligned}
$$

which are very close to equations (7) and (8). The slopes and intercepts of (9) and (10) yield more or less the same values of $h_{M e}$ and $\alpha$ as given by (7) and (8).

\section{Correlation of the HOMO and (HOMO-1)} energies of the indoles calculated by HF/STO-3G method with GT-calculated perturbational quantities

Since chemists are more familiar with the concept of molecular orbitals it is necessary to use how the GTcalculated perturbational quantities correlate with the HOMO. In table 5 the energies of HOMO and the next lower occupied molecular orbital (HOMO-1) of the indoles are given. These energies also correlate well with the perturbational quantities, the linear regression equations being

$$
\begin{aligned}
E_{\mathrm{HOMO}}= & (0.02315 \pm 0.00777) \sum_{r} C_{r 5}^{2} \\
& -(0.22349 \pm 0.00178) ; r=0.78
\end{aligned}
$$

Table 5. HOMO and HOMO-1 energies of the indoles calculated by HF/STO-3G method.

\begin{tabular}{lcc}
\hline System & $-E_{\mathrm{HOMO}} / \mathrm{ha}$ & $-\left(E_{\mathrm{HOMO}-1}\right) / \mathrm{ha}$ \\
\hline Indole & 0.22606 & 0.23942 \\
1-Methyl indole & 0.22063 & 0.23577 \\
2-Methyl indole & 0.21947 & 0.23484 \\
3-Methyl indole & 0.22004 & 0.23758 \\
1,2-Dimethyl indole & 0.21533 & 0.23056 \\
2,3-Dimethyl indole & 0.21420 & 0.23269 \\
2,5-Dimethyl indole & 0.21771 & 0.22651 \\
\hline
\end{tabular}

$$
\begin{aligned}
E_{\mathrm{HOMO}-1}= & (0.03021 \pm 0.00269) \sum_{r} C_{r 4}^{2} \\
& +(-0.23854 \pm 0.00054) ; r=0.98
\end{aligned}
$$

From these slopes and intercepts also we find that $h_{M e}$ and $\alpha$ have the same order of magnitude as obtained by the DFT results.

\section{Testing the reliability of the parameters $\alpha$ and $h_{M e}$ by correlating with CT transition energies of a series of $\mathrm{CT}$ complexes of the indoles with TCNE}

According to Mulliken's theory ${ }^{23,24}$ the CT transition energies are related to vertical ionization potentials $\left(I_{D}^{v}\right)$ of the donors by relation (13), where $C_{1}$ is given by eq. (14).

$$
h v_{C T}=I_{D}^{v}-C_{1}+\frac{C_{2}}{\left(I_{D}^{v}-C_{1}\right)}
$$

$$
C_{1}=E_{A}^{v}+G_{1}+G_{0} .
$$

Here $E_{A}^{v}$ is the vertical electron affinity of the acceptor, $G_{0}$ is the sum of several energy terms (like dipoledipole, van der Waals interaction, etc.) in the 'no-bond' state and $G_{1}$ is the sum of the number of energy terms in the 'dative' state. In most cases $G_{0}$ is small and can be neglected while $G_{1}$ is largely the electrostatic energy of interaction between the $D^{+}$and $A^{-}$. The terms $C_{2}$ in eq. (13) is related to the resonance energy of interaction

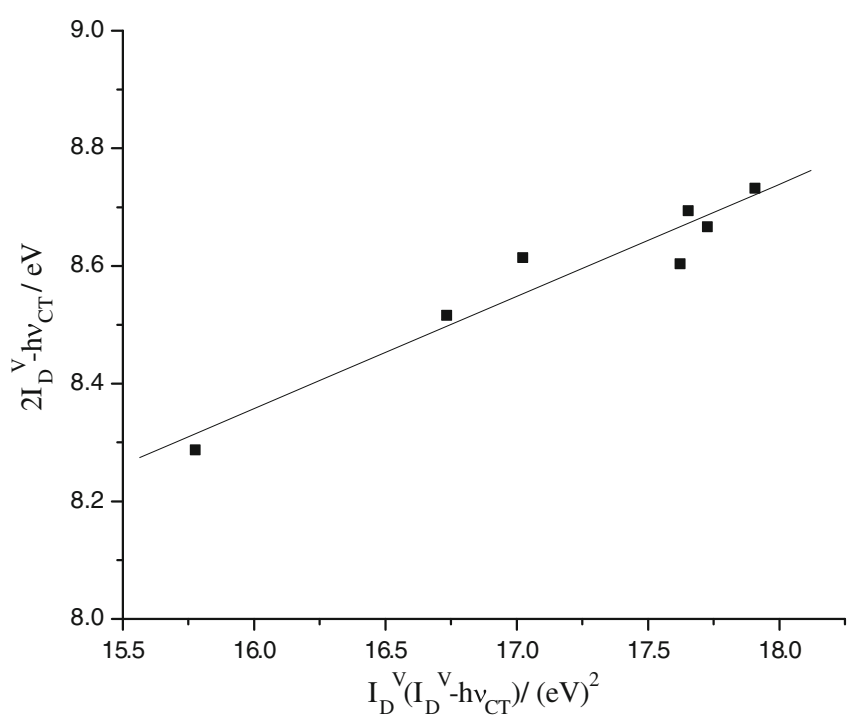

Figure 8. Plot according to equation (11) showing the validity of Mulliken's theory. 
Table 6. HOMO energies of the indoles re-calculated by the equation $E_{\text {Номо }}^{\prime}=\alpha+h_{N} \beta \sum_{r} C_{r j}^{2}$ using the presently found parameters $\alpha$ and $h_{M e}$ and experimental CT transition energies of complexes of the indoles with TCNE in dichloromethane medium.

\begin{tabular}{llc}
\hline Donor & $E_{\mathrm{HOMO}}^{\prime} / \mathrm{eV}$ & $h v_{C T} / \mathrm{eV}$ \\
\hline Indole & -5.5431346 & 2.472 \\
1-Methyl indole & -5.4400132 & 2.364 \\
2-Methyl indole & -5.46200605 & 2.230 \\
3-Methyl indole & -5.32293912 & 2.359 \\
1,2-Dimethyl indole & -5.35887277 & 2.051 \\
2,3-Dimethyl indole & -5.2418919 & 1.880 \\
2,5-Dimethyl indole & -5.44059404 & 2.149 \\
\hline
\end{tabular}

between the 'no-bond' and 'dative' forms in the ground and excited states and for a given acceptor it may be supposed constant. ${ }^{24} \mathrm{~A}$ rearrangement of eq. (13) yields eq. (15):

$$
2 I_{D}^{v}-h v_{C T}=\left(1 / C_{1}\right) I_{D}^{v}\left(I_{D}^{v}-h v_{C T}\right)+C_{1}+\frac{C_{2}}{C_{1}},
$$

$I_{D}^{v}$ is the negative of $E_{\mathrm{HOMO}}$ according to Koopmans theorem. ${ }^{42}$ Using the parameters $\alpha=-4.4$ and $h_{M e}=$ -0.25 the HOMO energies of the indoles were recalculated by eq. (1) and are re-designated as $E_{\mathrm{HOMO}}^{\prime}$. The CT transition energies $\left(h v_{C T}\right)$ of CT complexes of the indoles with TCNE in dichloromethane medium were collected from literature ${ }^{43}$ and are given in table 6 along with the re-calculated HOMO energies. Figure 8 shows an excellent linear correlation as expected from eq. (15), the regression equation being

$$
\begin{aligned}
2 I_{D}^{v}-h v_{C T}= & (0.19 \pm 0.02) I_{D}^{v}\left(I_{D}^{v}-h v_{C T}\right) \\
& +(5.30 \pm 0.41), r=0.96 .
\end{aligned}
$$

Such compliance with Mulliken's theory establishes the CT nature of the complexes.

\section{Conclusion}

In the present work it has been shown that graph theoretical methods streamline HMO-perturbation calculations through the use of simple theorems of polynomial algebra. The correlation of GT and DFT or Hartree-Fock methods yields the Coulomb integral $\alpha$ and the perturbational parameter $h_{M e}$ which quantifies the inductive effect of the methyl group attached to $\pi$ conjugated carbon atom. The value of $h_{M e}$ thus obtained is close to Sreitwieser's recommended value based on spectroscopic experiments. ${ }^{28}$

\section{Acknowledgements}

Authors are thankful to the learned reviewers for their valuable comments.

\section{References}

1. Hohenberg P and Kohn W 1964 Phys. Rev. B 136864

2. Kohn W and Sham L J 1965 Phys. Rev. A 1401133

3. Jena N K, Srinivasu K and Ghosh S K 2012 J. Chem. Sci. 124255

4. Srinivasu K and Ghosh S K 2011 J. Phys. Chem. C 115 1450

5. Ghosh C and Sarkar P 2007 J. Phys. Chem. Solids 68 1324

6. Mistri T, Dolai M, Chakroborty D, Khuda Bakshsh A R, Das K K and Ali M 2012 Org. Biomol. Chem. 102380

7. Webber B T, Per M C, Drumm D W, Hollenberg L C L and Russo S P 2012 Phys. Rev. B 8514102

8. Tiwary A S and Mukherjee A K 2008 J. Mol. StructTHEOCHEM 859107

9. Gupta A, Singh R P, Singh V B, Mishra B K and Sathyamurthy N 2007 J. Chem. Sci. 119457

10. Hückel E 1931 Z. Physik. 70204

11. Jursic B S 2000 J. Mol. Struct-THEOCHEM 498159

12. Hagebaum-Reignier D, Girardi R, Carissan Y and Humbel S 2007 J. Mol. Struct-THEOCHEM 81799

13. Dias J R and Guirgis G A 2002 Croat. Chim. Acta 75 621

14. Hall G G 1977 Mol. Phys. 33551

15. McClelland B J 1974 J. Chem. Soc. Farad. Tr. 701453

16. Sarkar J K and Mukherjee A K 1997 Mol. Phys. 90903

17. Dias J R 2004 Croat. Chim. Acta 77325

18. Dias J R 1987 Handbook of polycyclic hydrocarbons, Part A (1988 part B) (New York: Elsevier)

19. Dias J R and Aihara J 2009 Mol. Phys. 10771

20. Dias J R 2011 Open Org. Chem. J. 5112

21. Aihara J 2008 J. Phys. Chem. A 1124383

22. Coulson C A and Longuet-Higgins H C 1947 Proc. R. Soc. (London) A 19139

23. Mulliken R S 1952 J. Am. Chem. Soc. 74811

24. Mulliken R S 1955 J. Chem. Phys. 23397

25. Dias J R 1987 Can. J. Chem. 65734

26. Datta K and Mukherjee A K 1997 Int. J. Quant. Chem. 65199

27. Tiwary A S and Mukherjee A K 2008 Mol. Phys. 106 2271

28. Sreitwieser Jr. A 1961 Molecular orbital theory for organic chemists (New York: John Wiley), pp. 105

29. Demidovich B P and Marron I A 1976 Computational mathematics (Moscow: Mir), p. 176. See also Kurosh A G 1969 Higher Algebra (Moscow: Nauka)

30. Datta K K and Mukherjee A K 1989 Proc. Indian Acad. Sci. (Chem. Sci.) 101499

31. Ulam S 1960 A collection of mathematical problems, (New York: Wiley) p. 29

32. Becke A D 1993 J. Chem. Phys. 985648

33. Lee C, Yang W and Parr R G 1988 Phys Rev. B 37785

34. Frisch M J et al 2004 Gaussian 03, Revision D.01 (Wallingford CT: Gaussian, Inc.) 
35. Zhao Y and Truhler D G 2008 Theor. Chem. Acc. 120 215

36. Nijamudheen A, Jose D, Shine A and Datta A $2012 \mathrm{~J}$. Phys. Chem. Lett. 31493

37. Jissy A K, Ashik U P M and Datta A 2011 J. Phys. Chem. C $\mathbf{1 1 5} 12530$

38. Jose D and Datta A 2011 Cryst. Growth Des. 113137
39. Jissy A K and Datta A 2010 J. Phys. Chem B 114 15311

40. Frisch M J et al. 2009 Gaussian 09, Revision A.02: (Wallingford CT: Gaussian, Inc.)

41. Platt J R 1947 J. Chem. Phys. 15419

42. Koopmans T A 1933 Physica 1104

43. Foster R and Hanson P 1965 Tetrahedron 21255 